\title{
Phytotherapie in den medizinischen S3-Leitlinien der Arbeitsgemeinschaft der Wissenschaftlichen Medizinischen Fachgesellschaften - eine systematische Übersichtsarbeit
}

\author{
Petra Klose $^{\mathrm{a}}$ Karin Kraft $^{\mathrm{b}}$ Holger Cramer $^{\mathrm{a}}$ Romy Lauche ${ }^{\mathrm{a}}$ Gustav Dobos ${ }^{\mathrm{a}}$ \\ Jost Langhorst ${ }^{\mathrm{a}, \mathrm{c}}$ \\ ${ }^{a}$ Klinik für Naturheilkunde und Integrative Medizin, Kliniken Essen-Mitte, Medizinische Fakultät, Universität Duisburg Essen, Essen, \\ Deutschland

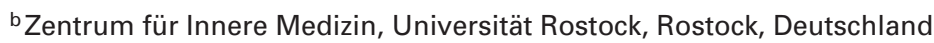 \\ ${ }^{c}$ Zentrum für Integrative Gastroenterologie, Kliniken Essen-Mitte, Medizinische Fakultät, Universität Duisburg Essen, Essen, Deutschland
}

\section{Schlüsselwörter}

Leitlinien · AWMF · Phytotherapie · Pflanzliche Arzneimittel · Medizinische Versorgung · Übersichtsarbeit

\section{Zuammenfassung}

In Deutschland werden an die interdisziplinär, evidenz- und konsensbasiert erstellten S3-Leitlinien der Arbeitsgemeinschaft der Wissenschaftlichen Medizinischen Fachgesellschaften (AWMF) höchste wissenschaftliche Ansprüche gestellt; sie prägen die Gestaltung der Patientenversorgung. Phytotherapeutika haben in der medizinischen Versorgung im deutschsprachigen Raum eine große Tradition. Zunehmend existiert Evidenz für ihre Wirksamkeit bei verschiedenen Erkrankungen. Die vorliegende Übersichtsarbeit untersucht, in welchem Ausmaß pflanzliche Arzneimittel in den aktuellen S3-Leitlinien Berücksichtigung finden. Mittels Überprüfung aller verfügbaren S3-Leitlinien der AWMF wurden die Empfehlungen/Statements zu phytotherapeutischen Therapieoptionen von 2 unabhängigen Experten identifiziert und in 3 Kategorien eingeteilt: positive, negative und offene. Darüber hinaus wurden Ausführungen zu Nebenwirkungen und Interaktionen recherchiert. In 40 $(31,3 \%)$ der 128 Leitlinien wird Phytotherapie benannt. In 55 Leitlinien $(42,9 \%)$, in denen grundsätzlich phytotherapeutische Therapieoptionen bestehen, werden pflanzlichen Arzneimittel nicht berücksichtigt. Von insgesamt 204 Empfehlungen zu Phytotherapeutika fallen $139(68,1 \%)$ negativ aus oder bleiben ergebnisoffen. Phytotherapie wird in 27 Leitlinien $(67,5 \%)$ im jeweiligen Kapitel zu komplementären und alternativen Verfahren und/oder unter Sonstiges eingeordnet, in 7 Leitlinien (17,5\%) steht sie am Ende der Empfehlungen zur medikamentösen Therapie, in 8 Leitlinien (20\%) zwischen den Empfehlungen zu konventionellen Verfahren. Hinweise zu Neben- und Wechselwirkungen finden sich zu 70 der 204 Empfehlungen $(34,3 \%)$. Weit weniger als die Hälfte der verfügbaren AWMF-S3-Leitlinien beinhalten eine Recherche zu Phytotherapeutika, nur in 17 S3-Leitlinien finden sich aktuell positive Empfehlungen. Eine systematische Aufarbeitung der vorhandenen Evidenz ist notwendig, um die adäquate Berücksichtigung der Phytotherapie in den medizinischen Leitlinien zu gewährleisten.

\section{Keywords}

Guidelines · Phytotherapy · Herbal medicinal products . Health care provision $\cdot$ Review

\section{Summary}

Phytotherapy in the German Medical AWMF S3 Guidelines a Systematic Overview

In Germany, the interdisciplinary, evidence- and consensus-based medical AWMF S3 guidelines represent the highest available scientific level and have significant impact on patient care. In Germany, Austria, and Switzerland, herbal medicine has a long tradition in medical care. Of notice, the evidence for its efficacy in numerous indications is increasing. This systematic review evaluates to which extent phytotherapy is taken into account in the current S3 guidelines. In all available medical AWMF S3 guidelines, the recommendations/statements on phytotherapeutic options were identified, and 2 independent experts classified them into 3 categories: positive, negative, and open. In addition, comments on side effects and interactions were extracted. Phytotherapeutic options are specified in $40(31.3 \%)$ out of 128 AWMF S3 guidelines. Phytotherapy was not included in 55 guidelines $(42.9 \%)$ with potential herbal therapeutic options. From 204 recommendations $139(68.1 \%)$ are negative or remain open. In 27 guidelines (67.5\%) phytotherapy is listed in the chapter for complementary and alternative medicine and/or miscellaneous, in 7 guidelines $(17.5 \%)$ it is mentioned at the end of the recommendations on medical drug therapy, and in 8 guidelines $(20.0 \%)$ it is listed in the recommendations regarding conventional treatments. Side effects and interactions are stated in 70 out of 204 recommendations (34.3\%). Less than half of the currently available medical AWMF S3 guidelines include phytotherapy in their search strategy. Only few positive statements and recommendations on herbal medicine are taken into account. A systematic and comprehensive work up of the existing evidence on the one hand and the consequent incorporation on the other is necessary to appropriately integrate phytotherapy in the German medical guidelines.

\section{KARGER}

Fax +497614520714

\section{(c) 2014 S. Karger AG, Freiburg}

$1661-4119 / 14 / 0216-0388 \$ 39.50 / 0$
Prof. Dr. Jost Langhorst

Kliniken Essen-Mitte, Knappschafts-Krankenhaus

Lehrstuhl für Naturheilkunde, Universität Duisburg-Essen

Am Deimelsberg 34a, 45276 Essen, Deutschland

J.Langhorst@kliniken-essen-mitte.de 


\section{Einleitung}

Die Pflanzenheilkunde ist eine der ältesten medizinischen Therapieformen weltweit und wird in allen Kulturen praktiziert. Sie ist zunächst eine auf Traditionen und Erfahrungen basierende Heilkunde. Die moderne, sogenannte rationale Phytotherapie folgt den Grundsätzen der wissenschaftlichen Medizin. Hierbei werden die Ausgangsstoffe und der Herstellungsprozess von Phytopharmaka standardisiert, die Indikationen exakt definiert und der Zusammenhang von Dosis und Wirkung wird als pharmakologisch charakterisierbar aufgefasst [1].

In der Phytotherapie werden entweder ganze Pflanzen, (häufiger) einzelne Teile (Blüte, Wurzel, Rinde, etc.), Bestandteile (ätherische Öle, etc.) oder Zubereitungen (Tinkturen, Tees, etc.) verwendet, die Krankheiten heilen, lindern oder vorbeugen sollen. Die einzelnen Pflanzenteile werden auch als pflanzliche Droge bezeichnet (z.B. Kamillenblüten, Baldrianwurzel, etc.). Homöopathische und anthroposophie Arzneimittel zählen aufgrund ihres besonderen Herstellungsprozesses nicht zu den Phytopharmaka [2].

Noch immer existieren Pauschalaussagen über Phytopharmaka wie z.B., dass «ihre therapeutische Wirksamkeit nicht oder nicht in ausreichendem Maße durch kontrollierte klinische Studien nachgewiesen worden ist, oder dass ihr NutzenRisiko-Verhältnis negativ bewertet wird» [3]. Dies entspricht jedoch nicht dem aktuellen wissenschaftlichen Kenntnisstand. Vielmehr sind Phytopharmaka in vielen Indikationsbereichen bereits umfangreich klinisch geprüft worden [4-7].

Diese Sichtweise wird auch durch die Arbeit der Europäischen Arzneimittelagentur EMA (European Medicines Agency) bestätigt [8]. Sie koordiniert die Bewertung und Überwachung aller Human- und Tierarzneimittel und spielt die zentrale Rolle in der europäischen Arzneimittelzulassung. Das Committee on Herbal Medicinal Products (HMPC) der EMA erarbeitet unter anderem umfassende Monografien für in der EU gebräuchliche Arzneidrogen und deren im Handel erhältliche Zubereitungen, die auf der Webseite offen zugänglich sind [9]. In diesen Monografien wird unter Zuhilfenahme eines öffentlichen Anhörungsprozesses der aktuelle Stand zur Wirksamkeit und Unbedenklichkeit von pflanzlichen Zubereitungen dokumentiert. Je nach Stand der wissenschaftlichen Evidenz wird der Status 'well-established use (WEU)' oder 'traditional use' erteilt. Für den WEU-Status muss wenigstens eine qualitativ gute klinische Studie vorliegen, wobei auch Negativstudien zu beachten sind. Die bisher geringe Anzahl der WEU-Positionen ist vor allem auf fehlende präklinische Sicherheitsdaten, z.B. zur Genotoxizität, zurückzuführen. WEU bedeutet die volle Akzeptanz hinsichtlich Wirksamkeit und Unbedenklichkeit für die in der Monografie genannten Indikationen. Arzneimittel mit WEU-Status sind in allen Ländern der EU zugelassen. Der Status 'traditional use' bedeutet dagegen, dass die entsprechende Zubereitung nicht für eine Indikation im Sinne des ICD-10 zugelassen wurde, da ihre Wirksam- keit nicht ausreichend belegt wurde. Wenn sie seit mindestens 30 Jahren, davon 15 in der EU, medizinisch verwendet worden ist, gilt das betreffende Arzneimittel als EU-weit registriert. Die Umwandlung vom traditionellen zum WEU-Status ist bei Vorlage entsprechender Daten stets möglich.

Vor diesem Hintergrund stellt sich die Frage, welchen Platz die moderne Phytotherapie im Kontext der evidenzbasierten Medizin einnimmt. Eine wichtige Rolle spielen bei dieser Beurteilung die medizinischen Leitlinien der Arbeitsgemeinschaft der Wissenschaftlichen Medizinischen Fachgesellschaften (AWMF), deren Hauptzweck in der Darstellung von Therapieempfehlungen auf der Basis von Studienlage und interdisziplinärem Expertenkonsens liegt.

Medizinische Leitlinien werden von der Deutschen Abteilung der Cochrane Collaboration, einem internationalen Netzwerk von Wissenschaftlern und Ärzten, das sich auf der Grundlage der evidenzbasierten Medizin die Verbesserung der wissenschaftlichen Grundlagen für Entscheidungen im Gesundheitssystem zur Aufgabe gemacht hat, wie folgt definiert: «Leitlinien (guidelines) sind systematisch entwickelte Aussagen zur Unterstützung der Entscheidungsfindung von Ärzten, anderen im Gesundheitssystem tätigen Personen und Patienten. Das Ziel ist eine angemessene gesundheitsbezogene Versorgung in spezifischen klinischen Situationen. Durch Leitlinien soll die Transparenz medizinischer Entscheidungen gefördert werden. Sie werden entwickelt, indem zu speziellen Versorgungsproblemen Wissen aus unterschiedlichen Quellen zusammengetragen und gewertet wird» [10].

In Deutschland hat sich die AWMF als Forum für Leitlinien etabliert. Die Leitlinien der AWMF werden entsprechend der Entwicklungsmethodik in 3 Klassen bzw. Stufen eingeteilt:

S1: von einer Expertengruppe im informellen Konsens erarbeitet (Ergebnis: Empfehlungen); S2: eine formale Konsensfindung (S2k) und/oder eine formale «Evidenz»-Recherche (S2e) hat stattgefunden; S3: Leitlinie mit allen Elementen einer systematischen Entwicklung (Logik-, Entscheidungsund «Outcome»-Analyse). Nationale Versorgungsleitlinien entsprechen methodisch der Klasse S3 [11].

Nur Leitlinien der Stufen 3 und S2e sind evidenzbasiert, ein systematisches und transparentes Vorgehen bei allen Schritten der Entwicklung ist hier entscheidend. In S3-Leitlinien werden Studienergebnisse auf ihre Wissenschaftlichkeit hin überprüft und je nach ihrer Qualität in verschiedene Evidenzklassen eingeteilt. Das Evidenzniveau richtet sich in der Mehrzahl nach den Oxford-Kriterien für evidenzbasierte Medizin [12] und wird einheitlich bewertet oder textlich umschrieben. In vielen Leitlinien werden darüber hinaus auch Behandlungsempfehlungen in unterschiedlicher Stärke festgelegt. Die Vergabe von Empfehlungsgraden wird durch die jeweiligen Leitlinienautoren im Rahmen eines formalen Konsensusverfahrens erarbeitet. Die Empfehlungen, vor allem deren Form, können sich daher von Leitlinie zu Leitlinie erheblich unterscheiden. 
In der vorliegenden Arbeit wird den Fragen nachgegangen, inwiefern sich die derzeitige Entwicklung einer zunehmenden wissenschaftlichen Aufarbeitung pflanzlicher Arzneimittel in der aktuellen Erstellung der deutschen S3-Leitlinien widerspiegelt, wo Lücken existieren und in welchen Bereichen Ergänzungsbedarf besteht.

\section{Methodik}

Die Leitliniensuche wurde am 15. März 2014 über die Internetseite der AWMF [13] durchgeführt, dazu wurden alle zu diesem Zeitpunkt verfügbaren Leitlinien identifiziert. Zudem wurden die parallel dazu veröffentlichten Leitlinienreporte identifiziert und für die Übersichtsarbeit herangezogen. Eingeschlossen wurden alle S3-Leitlinien der AWMF einschließlich der nationalen Versorgungsleitlinien.

Von 2 unabhängigen Untersuchern wurde von Hand überprüft, ob in diesen Leitlinien das Thema Phytotherapie bzw. pflanzliche Arzneimittel behandelt wurde. Da die Formulierungen für die jeweiligen Empfehlungen in den Leitlinien sehr unterschiedlich gestaltet sind, wurden im Rahmen der vorliegenden Arbeit die Empfehlungen für die in den einzelnen Leitlinien aufgenommenen Phytotherapeutika in 3 Kategorien zusammengefasst: 1) klar positive Empfehlungen, 2) klar negative und 3) neutral gefasste, offene Bewertungen, die ein Verfahren weder positiv noch negativ beurteilen (Tab. 1).

Die in den Leitlinien genannten Phytotherapeutika wurden aufgelistet und nach der Gesamtanzahl der Nennungen in allen verfügbaren Leitlinien sortiert. Informationen zu Wechsel- und Nebenwirkungen bzw. deren Fehlen, das Kapitel innerhalb der Leitlinie, in dem die Nennung erfolgte, sowie die bei der Nennung zitierte Literatur wurden ebenfalls durch 2 unabhängige Untersucher extrahiert. Zusätzlich wurden die Indikationen oder Symptome, falls genannt, gelistet. Die Ergebnisse beider Untersucher wurden miteinander verglichen und unterschiedliche Resultate diskutiert, um eine gemeinsame Beurteilung zu erzielen. In Zweifelsfällen wurde ein dritter Begutachter zurate gezogen.

Weiterhin wurden die Leitlinien hinsichtlich der Plausibilität der Nennung phytotherapeutischer Verfahren überprüft. Die Leitlinien wurden unterteilt in 1) Leitlinien, in denen phytotherapeutische Therapieoptionen potenziell vorhanden sind und 2) Leitlinien ohne potenzielle phytotherapeutische Therapieoptionen. Leitlinien der Kategorie 1 wurden gesondert bezüglich der Nennung phytotherapeutischer Therapieoptionen untersucht.

Zum Abschluss wurde eruiert, inwieweit Monografien und Berichte des HMPC bei der Leitlinienerstellung einbezogen worden sind. Hierzu wurden einerseits die in den Leitlinien aufgeführten pflanzlichen Drogen auf der Webseite der EMA recherchiert. Im Anschluss wurde ermittelt, ob Monografien vorliegen und welchen Status die einzelnen Zubereitungen erhalten haben [8]. Andererseits wurde in den jeweiligen Leitlinien nach Verweisen und Zitationen der ermittelten EMA-Monografien gesucht.

\section{Ergebnisse}

Insgesamt wurden 128 S3-Leitlinien auf der Internetseite der AWMF identifiziert. Von diesen waren zum Erhebungszeitpunkt 99 (77,3\%) gültig und 29 (22,7\%) formal abgelaufen. Alle 128 Leitlinien wurden in die Auswertung einbezogen.

\section{Empfehlungen}

Identifiziert wurden 40 Leitlinien $(31,3 \%)$, in denen phytotherapeutische Therapieoptionen begutachtet wurden. Sie sind in Tabelle 1 mit den dort aufgeführten Phytotherapeutika und den dazugehörigen Empfehlungen gelistet. In 2 Leitlinien wurden Arzneien ohne Empfehlung nur im Hinblick auf mögliche Nebenwirkungen aufgeführt.

Insgesamt wurden 204 Empfehlungen abgegeben: In 39 der 40 Leitlinien, die sich mit Phytotherapie auseinandergesetzt haben, wurden insgesamt 139 Empfehlungen für mehrheitlich deutsche Arzneipflanzen abgegeben. (In einer Leitlinie (Therapie der Adipositas im Kindes- und Jugendalter) hingegen gab es lediglich eine Empfehlung zu koreanischer Phytotherapie). Von diesen 139 Empfehlungen sind 45 positiv (32,4\%), 39 negativ $(28,1 \%)$ und 55 offen $(39,6 \%)$. Bei den Empfehlungen handelt es sich oftmals um Mehrfachnennungen derselben Pflanze (siehe unten), die bei diversen Krankheitsbildern zur Anwendung kommen kann.

In 14 Leitlinien wurden Kräuter oder Rezepturen aus der chinesischen Medizin genannt: Von den 21 Empfehlungen waren 4 positiv $(19,0 \%), 11$ negativ $(52,4 \%)$ und 6 offen $(28,6 \%)$ formuliert.

Aus den Kulturbereichen des Ayurveda, der japanischen und der koreanischen Medizin wurden insgesamt 9 Empfehlungen in 6 Leitlinien abgegeben: 4 sind positiv $(44,4 \%), 2$ negativ $(22,2 \%)$ und 3 offen $(33,3 \%)$.

Nahrungs- und Nahrungsergänzungsmittel (z.B. Früchte, Gewürze, Ballaststoffe etc.), die in Zusammenhang mit heilenden oder lindernden Wirkungen aufgeführt werden, finden sich in 10 Leitlinien mit 35 Empfehlungen: 12 positiv (34,3\%), 19 negativ $(54,3 \%)$ und 4 offen $(11,4 \%)$.

Bei den insgesamt 204 Empfehlungen fanden sich somit 65 positive (31,9\%), 71 negative $(34,8 \%)$ und 68 neutrale $(33,3 \%)$. Die 65 positiven Statements verteilen sich auf 17 der 128 Leitlinien (13,3\%).

\section{Nennungen}

Eine exakte Zählung der in den Leitlinien angegebenen bzw. genannten Phytotherapeutika findet sich in Tabelle 2. In 2 Leitlinien gibt es insgesamt 6 Aussagen zu pflanzlichen Arzneien, die keine Empfehlung beinhalten, sich nur auf Nebenbzw. Wechselwirkungen beziehen: «Halsschmerzen» (AWMF 053/010) und «Interdisziplinäre S3-Leitlinie für die Diagnostik, Therapie und Nachsorge des Mammakarzinoms» (AWMF 032 - 045OL).

Wurde Phytotherapie in einer Leitlinie beschrieben, so wurde im dazugehörigen Statement bzw. der Empfehlung am häufigsten der allgemeine Begriff Phytotherapie $(13 \times)$, chinesische Kräuter $(12 \times)$, Ayurveda $(4 \times)$ und japanische Medizin $(1 \times)$ gewählt: Dies macht 30 der 204 Empfehlungen aus. In den verbleibenden 174 Empfehlungen wurden insgesamt 92 unterschiedliche Arzneipflanzen und -kombinationen differenziert betrachtet, davon wurden 58 lediglich 1-mal genannt. 


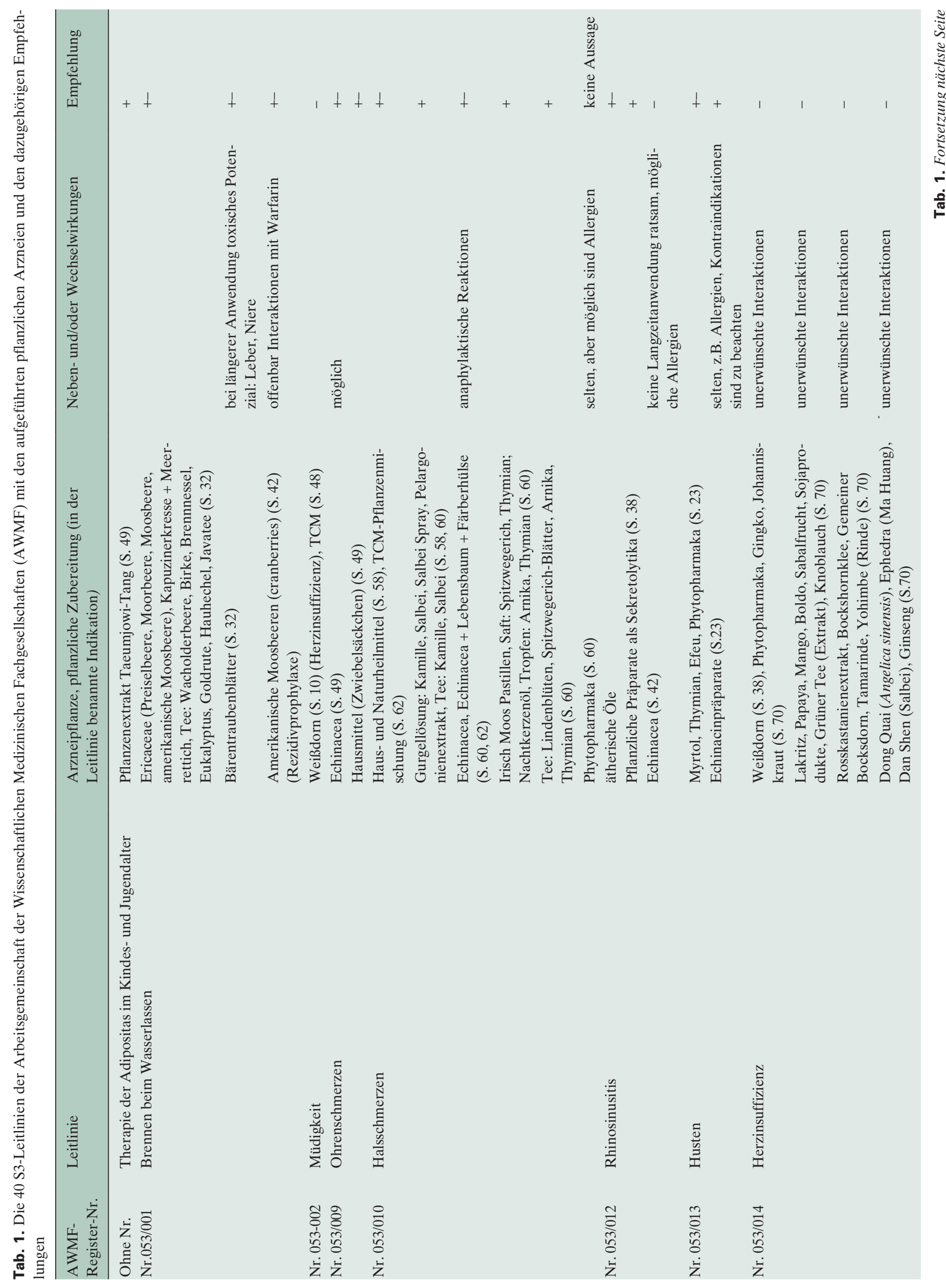




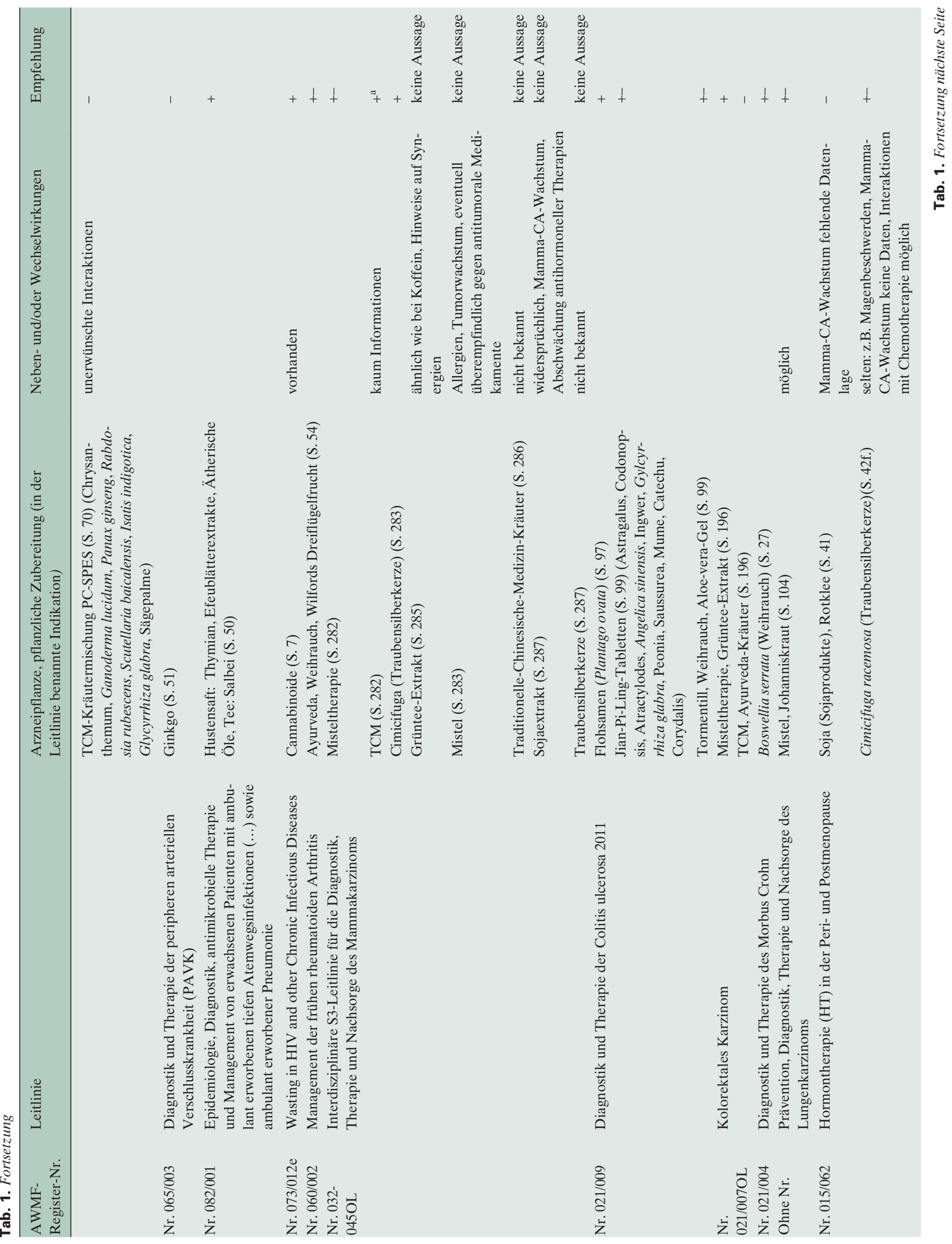




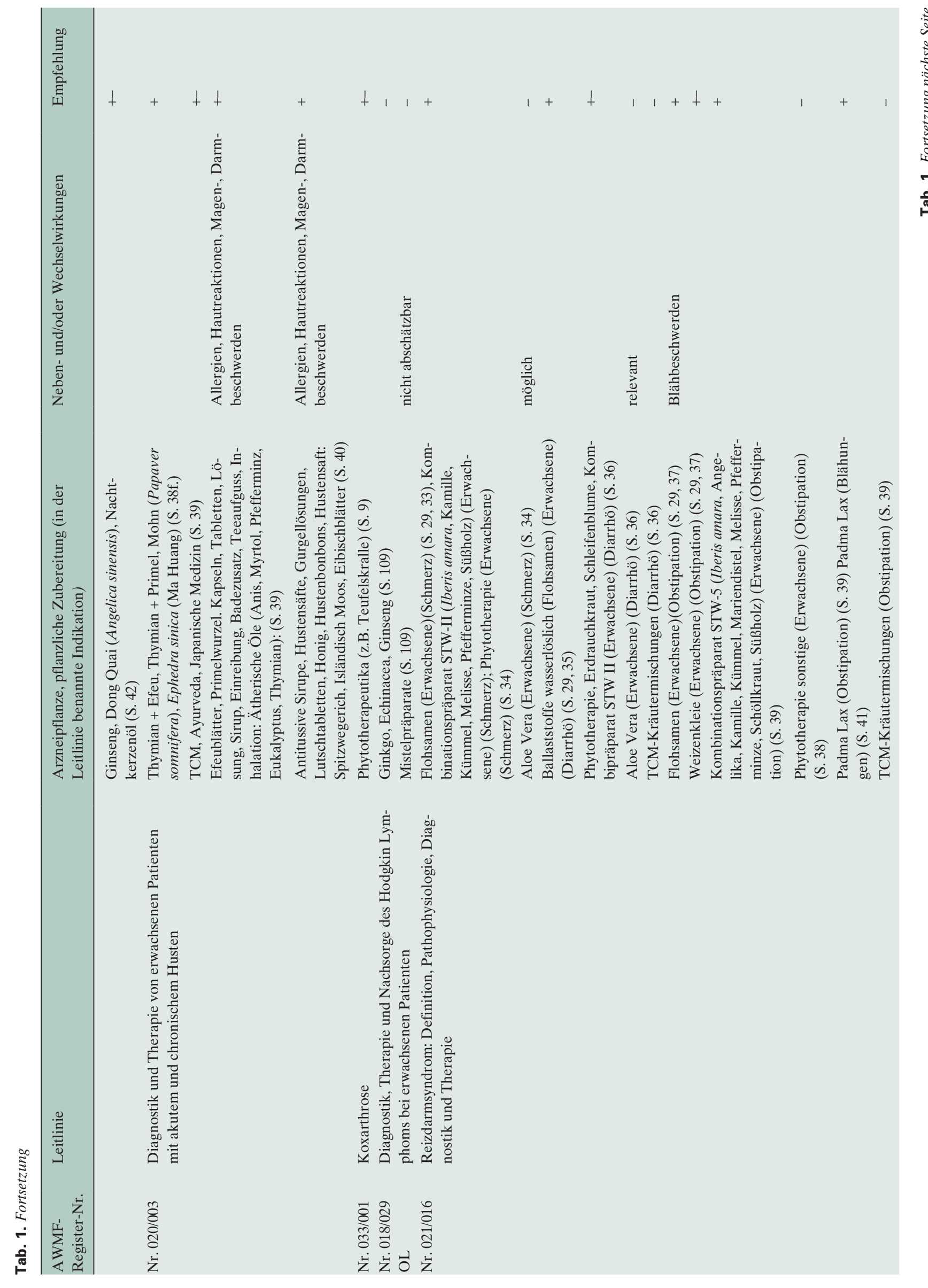




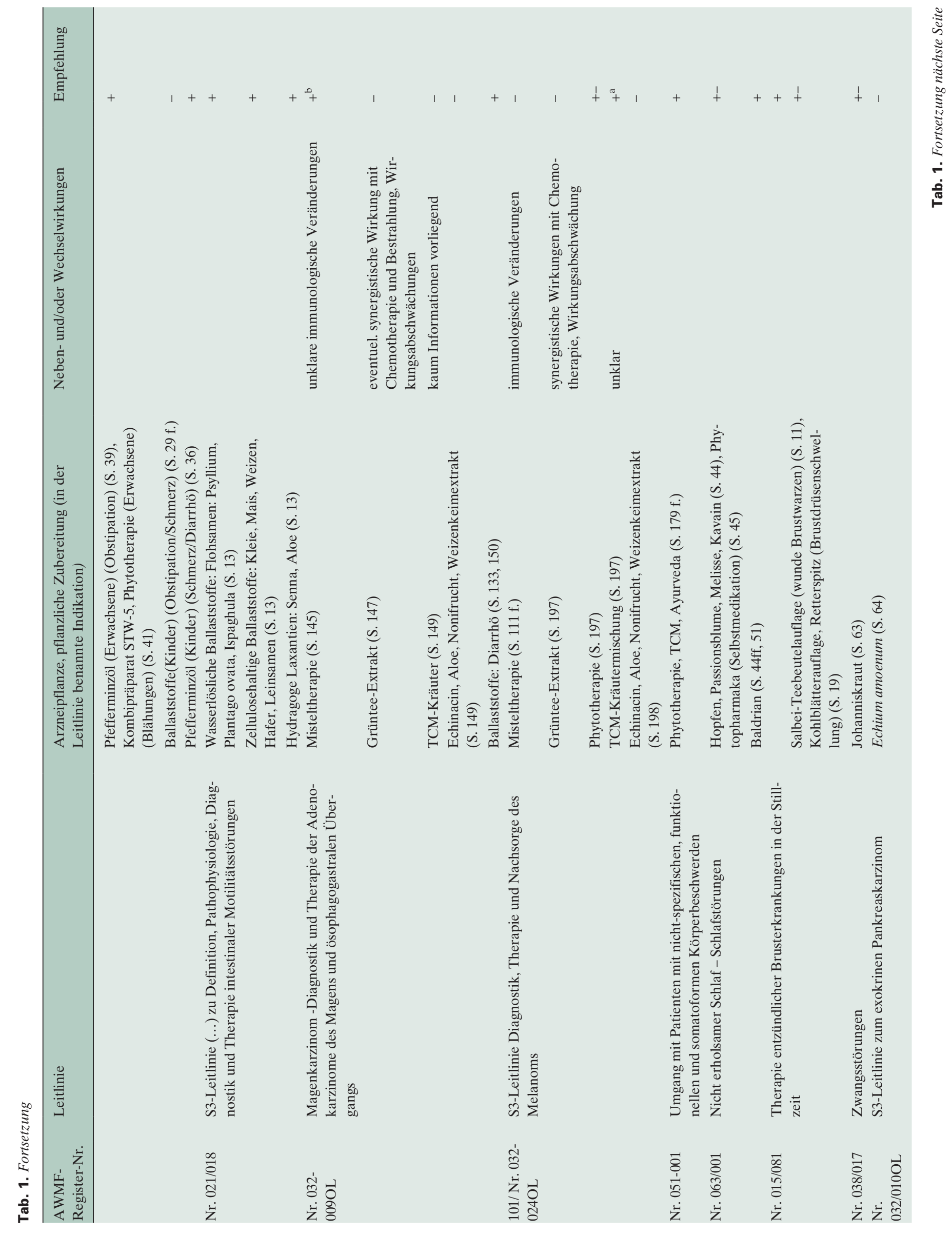




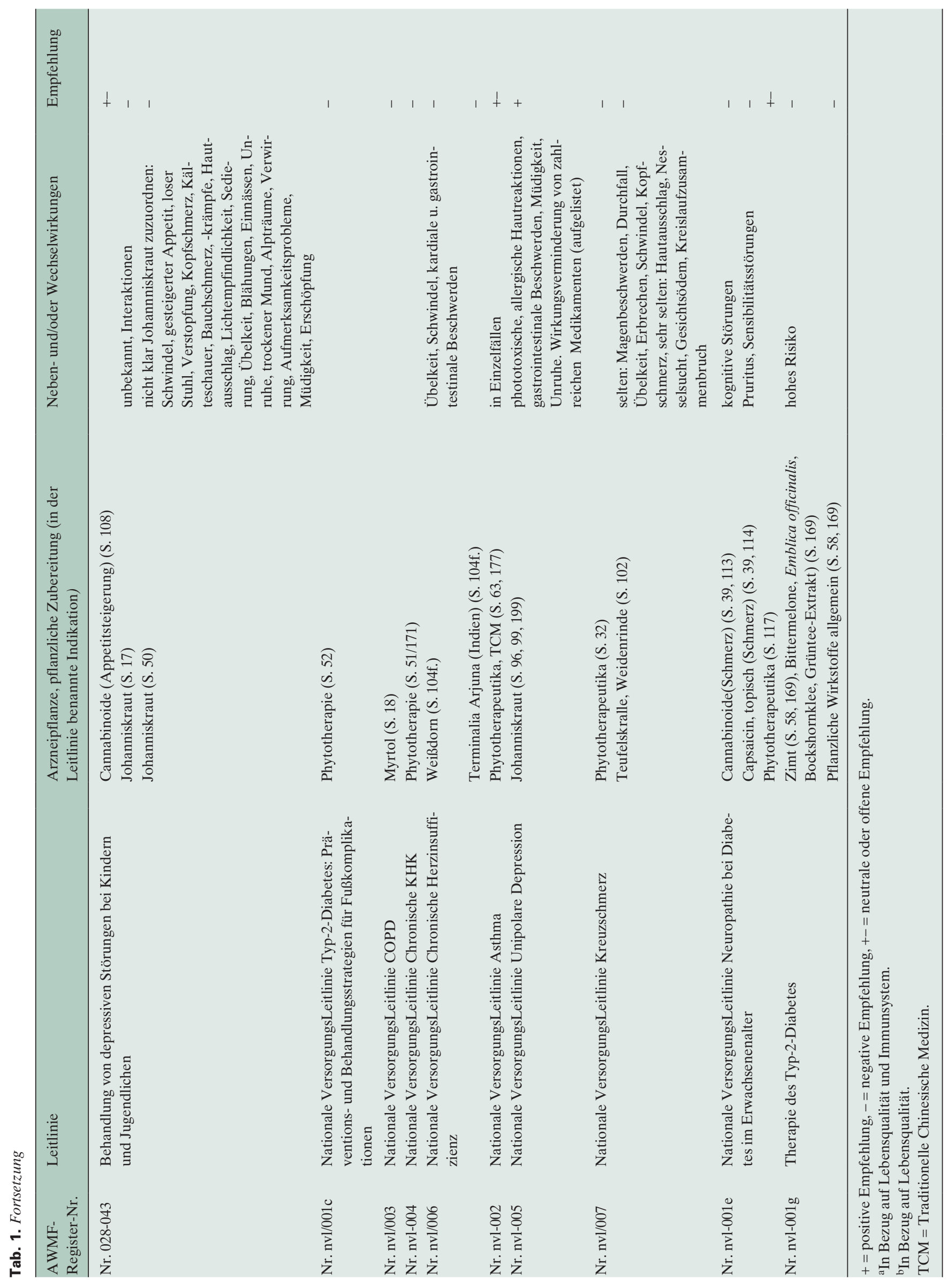


Tab. 2. Anzahl der Nennungen/Statements einzelner Phytopharmaka in den S3-Leitlinien

\begin{tabular}{|c|c|c|}
\hline $\begin{array}{l}\text { Anzahl } \\
\text { Statements }\end{array}$ & Heilpflanze(nteil)/Therapiesystem/Oberbegriff ${ }^{\mathrm{a}, \mathrm{b}}$ & $\begin{array}{l}\text { Anzahl } \\
\text { Pflanzen }\end{array}$ \\
\hline 13 & Phytotherapieb & $1^{\mathrm{b}}$ \\
\hline 12 & TCM bzw. Chinesische Kräuter ${ }^{b}$ & $1^{\mathrm{b}}$ \\
\hline 6 & $\begin{array}{l}\text { Johanniskraut, Mistel, Thymian, Echinacea, Aloe, Getreide als Ballaststoff (Mais, } \\
\text { Weizen, Hafer) }\end{array}$ & 6 \\
\hline 5 & Salbei, Flohsamen (diverse) & 2 \\
\hline 4 & Grüntee, Ayurveda ${ }^{\mathrm{b}}$ & $1+1^{b}$ \\
\hline 3 & $\begin{array}{l}\text { Pfefferminzöl, Cannabinoide, Weihrauch, Ginkgo, Ginseng, Spitzwegerich, Efeu, } \\
\text { Weißdorn, Soja, Myrtol, Ballaststoffe (allgemein) }{ }^{\mathrm{b}}\end{array}$ & $10+1^{b}$ \\
\hline 2 & $\begin{array}{l}\text { Kamille, Arnika, Nachtkerze, Ericaceae (Preiselbeere, Cranberry etc.), Bockshornklee, } \\
\text { Traubensilberkerze, Eukalyptus, Noni, Weizenkeime, Kleie, DongQuai, MaHuang, } \\
\text { Padma Lax, STW-5, STW-II, Ätherische Öle (allgemein) }{ }^{\mathrm{b}}\end{array}$ & $15+1^{b}$ \\
\hline 1 & $\begin{array}{l}\text { Bärentraube, Kapuzinerkresse/Meerrettich, Pelargonium, Irisch Moos, Thymian/Primel, } \\
\text { Thymian/Efeu, Efeu, Echinacea/Lebensbaum/Färberhülse, Rosskastanie, Tamarinde, } \\
\text { Rotklee, Bocksdorn, Yohimbe, Wilfords Dreiflügelfrucht, Tormentill, Mohn, Erdrauch- } \\
\text { kraut, Bittere Schleifenblume, Teufelskralle, Weidenrinde, Capsaicin, Hopfen, Baldrian, } \\
\text { Passionsblume, Eibisch, Primelwurzel, Melisse, Kavain, Indische Stachelbeere, Retterspitz, } \\
\text { Echium Amoneum, Isländisch Moos, Anis, Wacholder, Birke, Brennnessel, Goldrute, } \\
\text { Hauhechel, Javatee, Lindenblüten, Lakritz, Papaya, Mango, Knoblauch, Zwiebel, Boldo, } \\
\text { Sabalfrucht, Zimt, Bittermelone, Emblica officinalis, Leinsamen, Senna, Kohl, DanShen, } \\
\text { PC-SPES, JianPiLing, Taeumjowi-Tang, Terminalia Arjuna, Hausmittel (allgemein), } \\
\text { Traditionelle Japanische Medizin }{ }^{\mathrm{b}}\end{array}$ & $58+2^{b}$ \\
\hline \multicolumn{3}{|c|}{$\begin{array}{l}{ }^{a} \text { Verschiedene Darreichungsformen/Zubereitungen werden nicht getrennt aufgeführt. } \\
{ }^{b} \text { Therapiesysteme und Oberbegriffe werden auch gesondert (auf)gezählt. } \\
\text { TCM = Traditionelle Chinesische Medizin. }\end{array}$} \\
\hline
\end{tabular}

Bei den Empfehlungen zu den verschiedenen Arzneipflanzen weisen Johanniskraut, Mistel, Thymian, Echinacea, Aloe und Ballaststoffe aus Getreide jeweils 6 Statements auf, Salbei und Flohsamen jeweils 5 und Grüntee 4 Empfehlungen. 10 Phytotherapeutika (Tab. 2) wurden 3-mal, 15 Arzneipflanzen 2-mal begutachtet. Ballaststoffe allgemein wurden 3-mal, ätherische Öle 2-mal und Hausmittel 1-mal thematisiert.

\section{Nebenwirkungen}

In 22 der 40 Leitlinien (55\%) wurden Neben- und/oder Wechselwirkungen von Phytotherapie mit anderen Medikamenten oder Therapien aufgeführt (Tab. 1). Bei 70 (34,3\%) der insgesamt 204 Empfehlungen wurde auf diese Problematik eingegangen, und 6-mal wurden Neben- bzw. Interaktionen ohne Empfehlung vermerkt (siehe oben).

In 38 der Angaben zu Neben- und Wechselwirkungen finden sich unspezifische Aussagen wie: «nicht bekannt», «möglich», «unerwünschte Interaktionen», etc. In 38 Fällen sind die Angaben spezifisch («Allergien», «anaphylaktische Reaktionen», «toxisches Potenzial für Leber und Niere», «synergistische Wirkungen mit Chemotherapie, Wirkungsabschwächung», etc.) oder detailliert gelistet («Magenbeschwerden, Durchfall, Übelkeit, Erbrechen, Schwindel, Kopfschmerz», «Sehr selten: Hautausschlag, Nesselsucht, Gesichtsödem, Kreislaufzusammenbruch», usw.).

\section{Einordnung}

Bezüglich der inhaltlichen Aufteilung der Leitlinien zeigt sich, dass die Phytotherapie bei insgesamt 16 Leitlinien (40\%) in die Kategorie der «komplementären und/oder alternativen Verfahren» eingeordnet wurde. Bei 11 Leitlinien (27,7\%) stehen die phytotherapeutischen Therapieoptionen unter «Sonstiges» ganz am Schluss. In 7 Leitlinien (17,5\%) finden sich die Empfehlungen zur Phytotherapie am Ende des jeweiligen Kapitels der «Medikamentösen Therapie» (AWMF: 065/003 «Diagnostik und Therapie der peripheren arteriellen Verschlusskrankheit (PAVK)», 033/001 «Koxarthrose», nvl/003 «Nationale VersorgungsLeitlinie COPD», nvl-004 «Nationale VersorgungsLeitlinie Chronische KHK», nvl-005 «Nationale VersorgungsLeitlinie Unipolare Depression», nvl-007 «Nationale VersorgungsLeitlinie Kreuzschmerz», nvl-001e «Nationale VersorgungsLeitlinie: Neuropathie bei Diabetes im Erwachsenenalter»). In 8 Leitlinien (20,0\%) sind die Phytotherapeutika in die Leitlinie eingebettet, d.h. sie erscheinen hier im Kontext zwischen bzw. neben den konventionellen Empfehlungen (AWMF: 053-002 «Müdigkeit», 053/012 «Rhinosinusitis», 053/013 «Husten», 073/012e «Wasting in HIV and other Chronic Infectious Diseases», 020/003 «Diagnostik und Therapie von erwachsenen Patienten mit akutem und chronischem Husten», 021/016 «Reizdarmsyndrom: Definition, Pathophysiologie, Diagnostik und Therapie», 063/001 «Nicht erholsamer Schlaf - Schlafstörungen», 015/081 «Therapie entzündlicher Brusterkrankungen in der Stillzeit»). Werden 
Tab. 3. Leitlinien, in denen Phytotherapie bislang nicht vertreten ist, jedoch phytotherapeutische Therapieoptionen denkbar sind

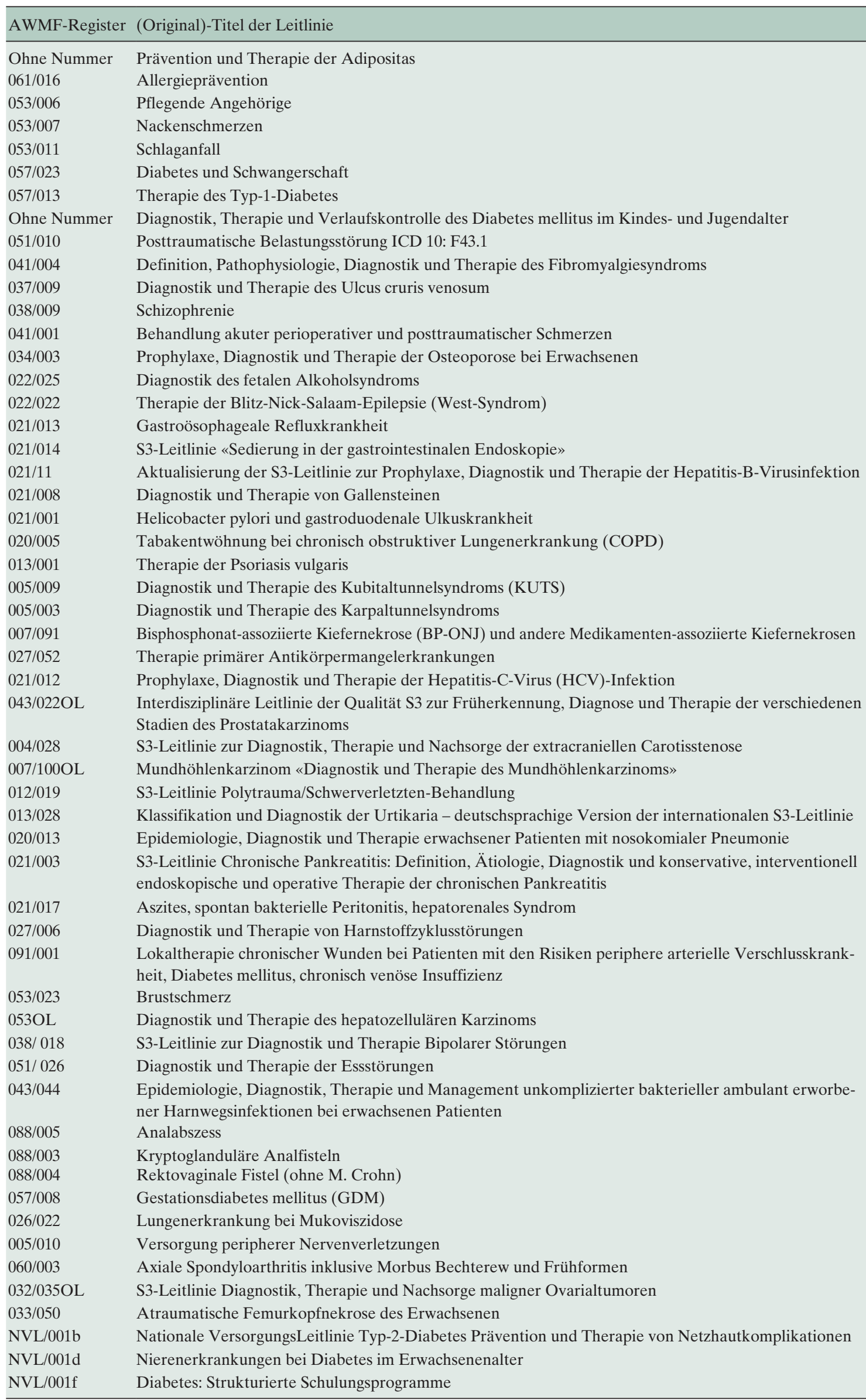


Tab. 4. Bewertungen der in den Leitlinien aufgeführten Phytotherapeutika durch die Europäische Arzneimittelagentur EMA

\begin{tabular}{lll}
\hline Zulassungsbereich & Arzneipflanze/pflanzliche Droge & Anzahl \\
\hline $\begin{array}{l}\text { Assessment report } \\
\text { (pre-final) }\end{array}$ & $\begin{array}{l}\text { Mistel, Angelica Sinensis (DangGui), Thymianöl, } \\
\text { 'Traditional use' }\end{array}$ & 8 \\
& Tormentill, Erdrauchkraut, Zwiebel, Salbeiöl, Bärentraube & \\
& $\begin{array}{l}\text { Zimt, Pelargonium, Ginseng, Passionsblume, Arnika, } \\
\text { Spitzwegerich, Thymian, Echinacea, Nachtkerze, }\end{array}$ & 40 \\
& Rosskastanienrinde, Bockshornklee, Curcumin, & \\
& Thymian/Primelwurzel, Anisfrucht, Anisöl, Isländisch & \\
& Moos, Eibisch, Hauhechel, Hafer, Hopfen, Melisse, & \\
& Teufelskralle, Bockshornklee, Primelwurzel, Primelblüten, & \\
& Pfefferminzblätter, Eukalyptusöl, Eukalyptusblätter, & \\
& Wacholder, Birke, Brennnesselwurzel, Brennesselblätter, & \\
& Goldrute, Lindenblüten, Lakritz, Weißdorn, Salbeiblätter, & \\
& Salbeiöl, römische Kamille, Grüner Tee & 9 \\
'Ttraditional +well- & Efeu, Rosskastaniensamen, Ginkgo, Aloe, Leinsamen, & 9 \\
established use' & Weidenrinde, Baldrian, Pfefferminzöl, Senna & \\
'Well-established use' & Traubensilberkerze, Capsaicin, Johanniskraut, Flohsamen & 4 \\
\hline
\end{tabular}

pflanzliche Therapieoptionen in verschiedenen Kapiteln genannt - dies ist in 2 Leitlinien der Fall (AWMF: 053/001 «Brennen beim Wasserlassen», 053-002 «Müdigkeit») - so wurde dies auch getrennt betrachtet.

\section{Literaturangaben}

In 5 Leitlinien (12,5\%) finden sich zu den aufgeführten Phytotherapeutika keine Literaturangaben, wobei in 4 dieser Leitlinien die Empfehlungen in die negative Kategorie fallen (AWMF: 065/003 «Diagnostik und Therapie der peripheren arteriellen Verschlusskrankheit (PAVK)», 082/ 001 «Epidemiologie, Diagnostik, antimikrobielle Therapie und Management von erwachsenen Patienten mit ambulant erworbenen tiefen Atemwegsinfektionen (...) sowie ambulant erworbener Pneumonie», 032-009OL «Magenkarzinom - Diagnostik und Therapie der Adenokarzinome des Magens und ösophagogastralen Übergangs», nvl/003 «Nationale VersorgungsLeitlinie COPD», nvl-001e «Nationale VersorgungsLeitlinie: Neuropathie bei Diabetes im Erwachsenenalter»). In 3 weiteren Leitlinien $(7,5 \%)$ wird nur ein Teil der angeführten Phytotherapeutika mit Studien belegt. Die entsprechenden Empfehlungen aus diesen Leitlinien gehören in alle 3 zuvor genannten Kategorien (AWMF: 015/062 «Hormontherapie (HT) in der Periund Postmenopause», 020/003 «Diagnostik und Therapie von erwachsenen Patienten mit akutem und chronischem Husten», 032 - 045OL «Interdisziplinäre S3-Leitlinie für die Diagnostik, Therapie und Nachsorge des Mammakarzinoms»).

\section{Leitlinien ohne Phytotherapie}

In 33 der 128 identifizierten Leitlinien $(25,8 \%)$ sind pflanzliche Arzneimittel aus Plausibilitätsgründen keine Therapieoption. Beispielhaft seien die folgenden 3 Leitlinien genannt: «Chirurgie der Adipositas» (AWMF Register 088/001), «S3Leitlinie zur intensivmedizinischen Versorgung herzchirurgischer Patienten: Hämodynamisches Monitoring und HerzKreislauf« (AWMF Register 001/016) oder «Leitlinie Fissuren- und Grübchenversiegelung» (AWMF Register 083/002).
Bei Durchsicht der 128 S3-Leitlinien wurden neben den 40 Leitlinien, die Phytotherapie aufführen, weitere 55 Leitlinien $(42,9 \%)$ identifiziert, in denen phytotherapeutische Optionen bestehen, die aber nicht berücksichtigt wurden (Tab. 3).

\section{Vergleich mit EMA-Monografien}

$\mathrm{Zu}$ den 92 in den Leitlinien genannten Phytotherapeutika finden sich auf der Webseite der EMA 53 finale und 8 präfinale Monografien [14]. In den finalen Monografien wurden 40 pflanzliche Drogen mit 'traditional use' $(75,5 \%)$ bewertet, 9 erhielten je nach Evidenzlage der jeweiligen Zubereitungen die Bewertungen 'traditional use' und WEU (17,0\%) und 4 pflanzliche Drogen den WEU-Status (7,5\%) für die jeweils dort angegebene Indikation (Tab. 4).

\section{Diskussion}

\section{Zusammenfassung der Ergebnisse}

In dieser Arbeit wurden die aktuellen Angaben zur Abbildung und Bewertung von Phytotherapeutika in den medizinischen S3-Leitlinien der AWMF mit folgenden Resultaten analysiert:

In 40 Leitlinien wurde das Thema Phytotherapie behandelt. In knapp der Hälfte der Leitlinien wurde Phytotherapie unter der Rubrik «komplementäre und alternative Verfahren» abgehandelt, in 8 der 40 Leitlinien hat die Phytotherapie entsprechend den anderen Therapieoptionen ein eigenes Kapitel erhalten, in 7 weiteren wurde sie unter «medikamentöse Therapien» subsumiert. In 8 Leitlinien wurde zwar das Thema Phytotherapie behandelt, die Aussagen sind jedoch nicht durch Referenzen belegt. Bei 55 Leitlinien ohne Hinweise auf Phytotherapie wäre die Aufnahme von Phytotherapie als Therapieoption denkbar gewesen.

Insgesamt wurden 92 verschiedene Phytotherapeutika aufgeführt. Jeweils ca. ein Drittel der zugehörigen Empfehlungen ist positiv, negativ oder offen. Weniger als die Hälfte der 
Leitlinien widmen sich dem Thema «Nebenwirkungen und Wechselwirkungen von Phytotherapeutika». Diese sind wiederum in weniger als der Hälfte spezifisch und konkret. In keiner einzigen Leitlinie wurde das umfangreiche wissenschaftliche Material der Monografien des HMPC berücksichtigt.

\section{Interpretation}

Die Ergebnisse dieser Arbeit weisen darauf hin, dass das Thema Phytotherapie in den Leitlinien der AWMF sehr unterschiedlich behandelt wird. Während in einigen Leitlinien ausführlich Stellung bezogen wird, werden andere Empfehlungen und Statements vor allem aus systematischen Übersichtsarbeiten und/oder klinischen Studien abgeleitet. In wieder anderen wird das Thema eher pragmatisch behandelt, d.h. es werden Phytotherapeutika empfohlen, für deren Einsatz keine ausreichende Evidenz vorliegt, die jedoch auf eine lange Anwendungstradition zurückgreifen können. Einige Leitlinien verzichten ganz oder teilweise auf Begründungen oder Literaturverweise, und in über einem Drittel aller S3-Leitlinien werden phytotherapeutische Therapieoptionen, obgleich wahrscheinlich vorhanden, nicht überprüft und bewertet.

\section{Nutzung von Phytotherapie}

Die Wichtigkeit und Dringlichkeit, Phytotherapie wissenschaftlich fundiert in den deutschen Leitlinien zu begutachten, ist evident - dies unterstützen auch aktuelle Zahlen zum Gebrauch von Phytopharmaka. In einer von 2007 bis 2008 durchgeführten Erhebung zum Gebrauch pflanzlicher Arzneimittel bei Kindern in Deutschland [15] zeigte eine Elternbefragung, dass $85,5 \%$ der Kinder Phytopharmaka erhalten hatten. Auch als in Deutschland nach Einstellung der Erstattungsfähigkeit für die meisten Phytopharmaka, deren ärztliche Verordnung zwischen 2004 und 2005 stark zurückging [16], sank der Verbrauch der in Selbstmedikation angewendeten pflanzlichen Arzneimittel zunächst nur leicht und war im Jahr 2013 mit 1,1 Milliarden EUR Umsatz praktisch wieder auf dem Stand von 2002 angekommen [17].

Eine Erhebung in Großbritannien [18] im Jahr 2005 ergab, dass die Inanspruchnahme pflanzlicher Arzneimittel in den vorangegangenen 12 Monaten an vierter Stelle von 23 komplementären Therapieoptionen stand. Die Prävalenz der Nutzung pflanzlicher Arzneien erreicht bei verschiedenen Krankheitsbildern hohe Werte. So nutzen nach einer US-amerikanischen Studie 40\% der Patientinnen mit Ovarialkarzinom während der Chemotherapie mindestens ein pflanzliches Mittel [19], bei Erkrankungen des rheumatischen Formenkreises sind es mitunter bis zu 90\% [20].
Bei pflanzlichen Arzneimitteln muss wie bei allen anderen Arzneimitteln der Sicherheitsaspekt berücksichtigt werden, d.h. das Potenzial für unerwünschte Arzneimittelwirkungen, Kontraindikationen und Arzneimittelinteraktionen [21]. Insbesondere beim Einsatz zur Linderung von Symptomen bei schweren chronischen oder lebensbedrohlichen Erkrankungen, für die diese Mittel in hohem Maße eingenommen werden [19], aber ebenso bei leichten Erkrankungen sind verlässliche Informationen zu Wirksamkeit und Sicherheit für Ärzte [22] und Anwender [23] unerlässlich.

\section{Fehlende Thematisierung der Phytotherapie}

In 55 Leitlinien wurde das Thema Phytotherapie nicht erwähnt, obwohl eine Anwendung von Phytotherapeutika bei den entsprechenden Erkrankungen durchaus denkbar ist. Als mögliche Ursachen zu diskutieren sind, dass phytotherapeutische Optionen nicht in Erwägung gezogen bzw. recherchiert wurden oder dass deren Evidenz gesichtet, jedoch als nicht ausreichend eingestuft wurde und eine schriftliche Darlegung in der Leitlinie deshalb nicht in Betracht gezogen wurde.

\section{Schlussfolgerung}

Eine systematische Aufarbeitung der Evidenz zu Phytotherapie im Rahmen der Leitlinienerstellung erscheint angesichts der hohen Prävalenz ihrer Nutzung dringend geboten, um Behandlern und Patienten gesicherte Informationen über Wirksamkeit und Sicherheit zu liefern, die für eine erfolgreiche Behandlung unerlässlich sind. Eine Mitarbeit von Experten auf dem Gebiet der Phytotherapie ist dabei hilfreich und deshalb anzustreben. Mit der Aufnahme der Gesellschaft für Phytotherapie [24] in die AWMF Anfang November 2013 besteht nun die Gelegenheit, das Thema Phytotherapie in medizinischen Leitlinien systematisch aufzuarbeiten.

\section{Dank}

Die Arbeit wurde von der Rut- und Klaus-Bahlsen-Stiftung gefördert.

\section{Disclosure Statement}

Die Autoren erklären hiermit, dass keine Interessenskonflikte in Bezug auf die vorliegende Arbeit bestehen. 


\section{Literatur}

1 Kraft K, März R: Die wissenschaftliche Basis der Phytotherapie. Zeitschrift für Phytotherapie 2006; 27:279-283.

2 Schilcher H, Kammerer S, Wegener T: Leitfaden Phytotherapie. München, Elsevier, 2010, pp 2-4.

$\checkmark 3$ Schwabe U: Arzneiverordnungen 2012 im Überblick; in Schwabe U, Paffrath D (eds): Arzneiverordnungs-Report 2013: Aktuelle Daten, Kosten, Trends und Kommentare. Berlin, Springer, 2013, pp 39-41.

-4 Kumar S, Ahuja V, Sankar MJ, Kumar A, Moss AC: Curcumin for maintenance of remission in ulcerative colitis. Cochrane Database Syst Rev 2012; 10:CD008424.

5 Rambaldi A, Jacobs BP, Gluud C: Milk thistle for alcoholic and/or hepatitis $\mathrm{B}$ or $\mathrm{C}$ virus liver diseases. Cochrane Database of Syst Rev 2007; 4:CD003620.

6 Mason L, Moore RA, Derry S, Edwards JE, McQuay HJ: Systematic review of efficacy of topical rubefacients containing salicylates for the treatment of acute and chronic pain. BMJ 2004;328:991.

7 Fritz H, Seely D, McGowan J, Skidmore B, Fernandes R, Kennedy DA, Cooley K, Wong R, Saga S, Balneaves LG, Fergusson D: Black cohosh and breast cancer: a systematic review. Integr Cancer Ther 2013;12:7-24.

8 European Medicines Agency (EMA). www.ema. europa.eu (Zugriff 25.11.14).

9 Definition. Committee on Herbal Medicinal Products. www.ema.europa.eu/ema/index.jsp?curl=pages/ regulation/general/general_content_000208.jsp (Zugriff 25.11.14).
10 Deutsche Abteilung der Cochrane Collaboration. www.cochrane.de/de/leitlinien (Zugriff 25.11.14)

11 Ärztliches Zentrum für Qualität in der Medizin. Leitlinien. www.leitlinien.de/leitlinienmethodik/leitlinienglossar/glossar/klassifizierung-von-leitlinien (Zugriff 25.11.14).

12 Centre for Evidenced-Based Medicine. www.cebm. net/oxford-centre-evidence-based-medicine-levelsevidence-march-2009/ (Zugriff 25.11.14).

13 Arbeitsgemeinschaft der Wissenschaftlichen Medizinischen Fachgesellschaften (AWMF). www. awmf.org (Zugriff 25.11.14).

14 European Medicines Agency (EMA), Pflanzliche Arzneien- Monografien. http://www.ema.europa.eu/ ema/index.jsp?curl=pages/includes/medicines/medicines_landing_page.jsp\&mid $=($ Zugriff 25.11.14).

15 Hümer M, Scheller G, Kapellen T, Gebauer C, Schmidt H, Kiess W: Use of herbal medicine in German children - prevalence, indications and motivation. Dtsch Med Wochenschr 2010;13:959-964.

16 Linde K, Alscher A, Friedrichs C, Joos S, Schneider A: The use of complementary and alternative therapies in Germany - a systematic review of nationwide surveys. Forsch Komplementmed 2014; 21:111-118.

17 Bundesverband der Arzneimittelhersteller e.V.: Der Arzneimittelmarkt in Deutschland in ZahlenVerordnungsmarkt und Selbstmedikation. 2013; Kapitel 9. https://www.bah-bonn.de/index.php?eID= dumpFile \& $t=f \& f=4089 \&$ token $=3 e c 3 e b 2533 e 1 c 4 c 5 a$ b6a42e783f0651d200c7e08 (Zugriff 25.11.14).
8 Hunt KJ, Coelho HF, Wider B, Perry R, Hung SK, Terry R, Ernst E: Complementary and alternative medicine use in England: results from a national survey. Int J Clin Pract 2010;64:1496-1502.

19 Andersen MR, Sweet E, Lowe KA, Standish LJ, Drescher CW, Goff BA: Dangerous combinations: ingestible CAM supplement use during chemotherapy in patients with ovarian cancer. J Altern Complement Med 2013;19:714-720.

20 Herman CJ, Allen P, Hunt WC, Prasad A, Brady TJ: Use of complementary therapies among primary care clinic patients with arthritis. Prev Chronic Dis 2004;1:A12.

21 Izzo AA, Ernst E: Interactions between herbal medicines and prescribed drugs: an updated systematic review. Drugs 2009;69:1777-1798.

22 Trimborn A, Senf B, Muenstedt K, Buentzel J, Micke O, Muecke R, Prott FJ, Wicker S, Huebner J: Working Group Prevention and Integrative Oncology of the German Cancer Society. Attitude of employees of a university clinic to complementary and alternative medicine in oncology. Ann Oncol 2013;24:2641-2645.

23 Broom A, Tovey P: The role of the Internet in cancer patients' engagement with complementary and alternative treatments. Health (London) 2008;12: 139-155.

24 Deutsche Gesellschaft für Phytotherapie. www. phytotherapy.org (Zugriff 25.11.14) 